\title{
Preparation, Stabilisation and Dissolution Enhancement of Vortioxetine Hydrobromide Metastable Polymorphs in Silica \\ Nanopores
}

Yun Cao a, Keke Zhang a, Zhenguo Gao a *, Jingkang Wang a, b, Sohrab Rohani ${ }^{\text {c, Junbo }}$ Gong $^{a, b, d}$

${ }^{\text {a }}$ School of Chemical Engineering and Technology, State Key Laboratory of Chemical Engineering, Tianjin University, Tianjin 300072, China

${ }^{\mathrm{b}}$ Key Laboratory Modern Drug Delivery and High Efficiency in Tianjin, P. R. China

${ }^{c}$ Department of Chemical and Biochemical Engineering, The University of Western Ontario, London, Ontario N6A 5B9, Canada

d Chemistry and Chemical Engineering Guangdong Laboratory, Shantou 515031, P. R. China

* Tel: +86-22-27403200. E-mail: zhenguogao@tju.edu.cn. 


\section{Comparison of PXRD patterns of blank silica particles with $3 \mathbf{~ n m}$ pore sizes}

and its composite with amorphous VH

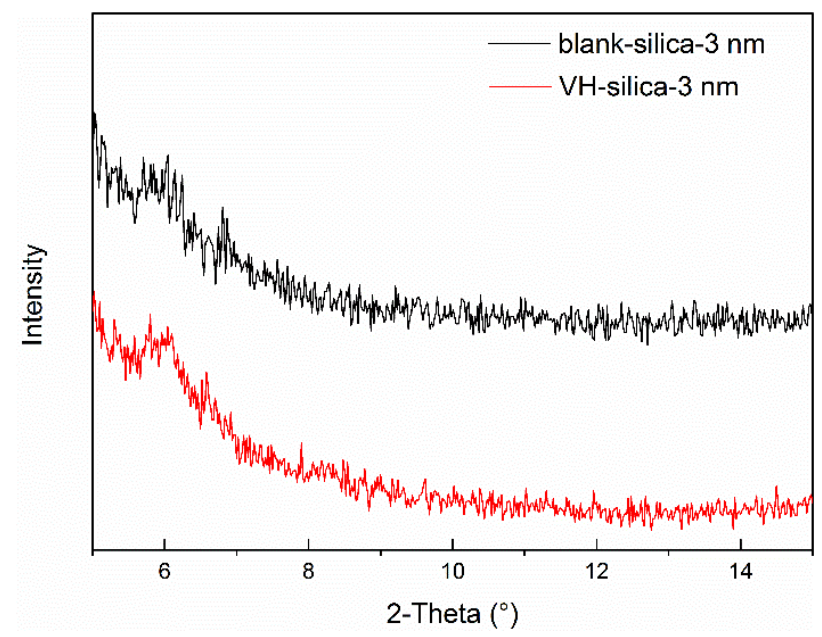

Figure S1. PXRD patterns of blank silica particles with $3 \mathrm{~nm}$ pore sizes and their composite with amorphous $\mathrm{VH}$.

2. Solid state of bulk form $\alpha$ and confined form $\alpha$ during physical stability experiments under different temperature-humidity conditions.
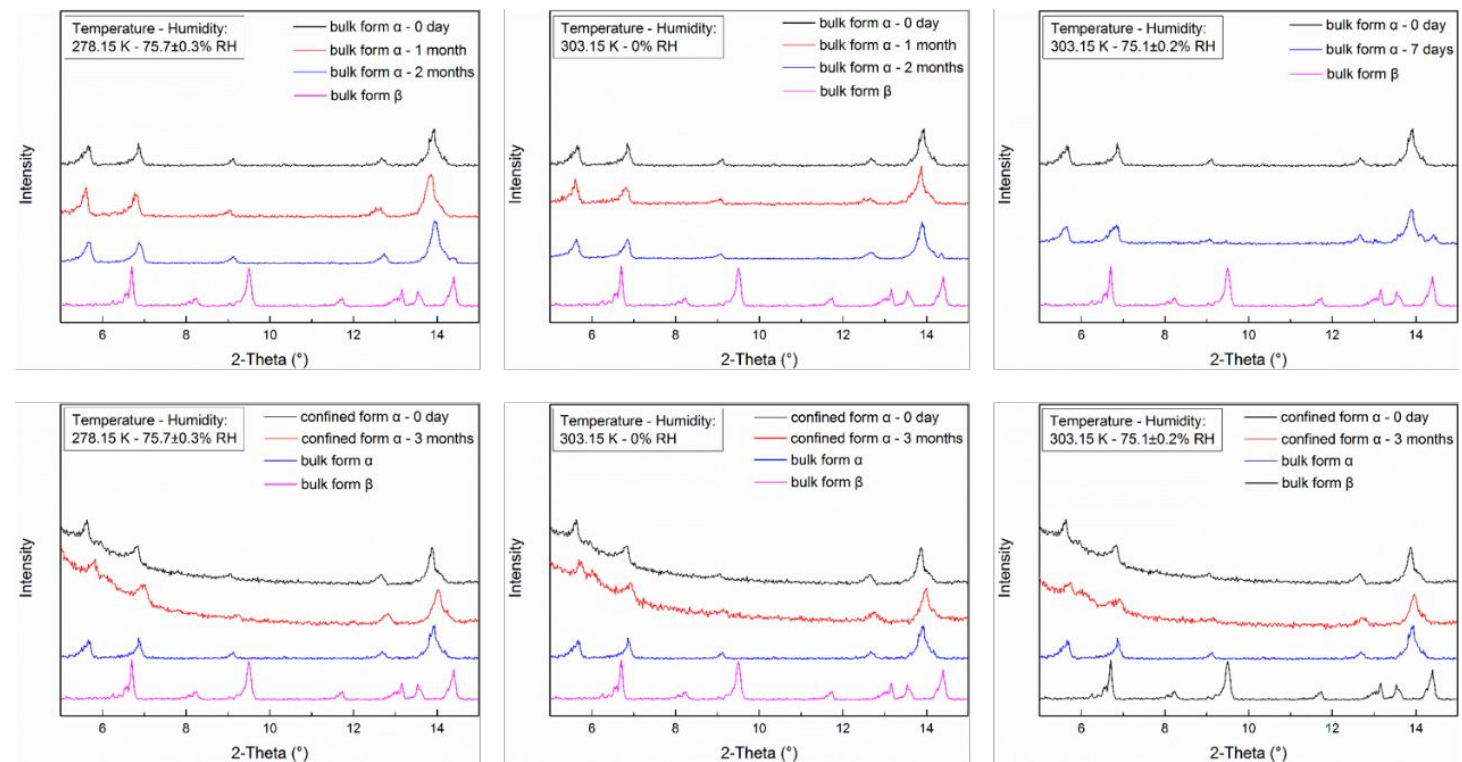

Figure S2. Physical stability of bulk form $\alpha$ and confined form $\alpha$ in different temperature-humidity conditions.

3. Determination of form $\alpha$ solubility in phosphate buffer (pH 6.8) at $310.15 \mathrm{~K}$.

The $\mathrm{VH}$ form $\alpha$ is the metastable form and can easily convert to the stable form $\beta$ 
in solution. The solubility of form $\alpha$ in phosphate buffer (pH 6.8) at $310.15 \mathrm{~K}$ was determined by the dynamic method. ${ }^{1}$ A finite amount (1.5-3.00 mg) of form $\alpha$ was added into $300 \mathrm{~mL}$ of phosphate buffer ( $\mathrm{pH}$ 6.8) until dissolved completely. A thermostat (model CF41, Julabo GmbH, Germany) was employed to control temperature. Then, form $\alpha$ was added to the clear solution repeatedly until the particles remained suspended for about $10 \mathrm{~min}$ and the particle counts did not decrease significantly. At this time, form $\alpha$ would not dissolve, and the system reached the dissolution equilibrium. PXRD was used to identify the polymorphs of suspended particles. Results showed that no polymorphic conversion had happened in this experiment. The experiment was repeated three times. The solubility of form $\alpha$ was equal to the average dissolved amount in saturated solution (Table S1).

Table S1. VH form $\alpha$ solubility in phosphate buffer (pH 6.8) at $310.15 \mathrm{~K}$

\begin{tabular}{lllll}
\hline & $\begin{array}{l}\text { Volume of phosphate } \\
\text { buffer } / \mathrm{mL}\end{array}$ & $\begin{array}{l}\text { Amount of form } \alpha \\
\text { added } / \mathrm{mg}\end{array}$ & $\begin{array}{l}\text { Average amount of } \\
\text { form } \alpha \text { added } / \mathrm{mg}\end{array}$ & $\begin{array}{l}\text { Form } \alpha \text { solubility } \\
/(\mathrm{mg} / \mathrm{mL})\end{array}$ \\
\hline 1 & 300 & 34.29 & 33.98 & 0.1133 \\
2 & 300 & 33.87 & & \\
3 & 300 & 33.78 & & \\
\hline
\end{tabular}

\section{Crystal structure information of VH form $\beta$.}

Table S2. crystal structure information of $\mathrm{VH}$ form $\beta$

\begin{tabular}{ll}
\hline Crystal data & VH form $\beta$ \\
\hline Crystal system, space group & $\mathrm{P}{ }_{1} / \mathrm{c}(14)$ \\
Temperature $(\mathrm{K})$ & 150 \\
$a, b, c(\AA)$ & $a 13.000(4) b 18.213(3) c 7.7621(16)$ \\
$\alpha, \beta, \gamma\left({ }^{\circ}\right)$ & $\alpha 90^{\circ} \beta 100.844^{\circ} \gamma 90^{\circ}$ \\
$V\left(\AA^{3}\right)$ & 1805.01 \\
$Z, Z^{\prime}$ & 4,1 \\
\hline
\end{tabular}

5. Solid-state of confined form $\alpha$ under methyl acetate vapor for one month. 

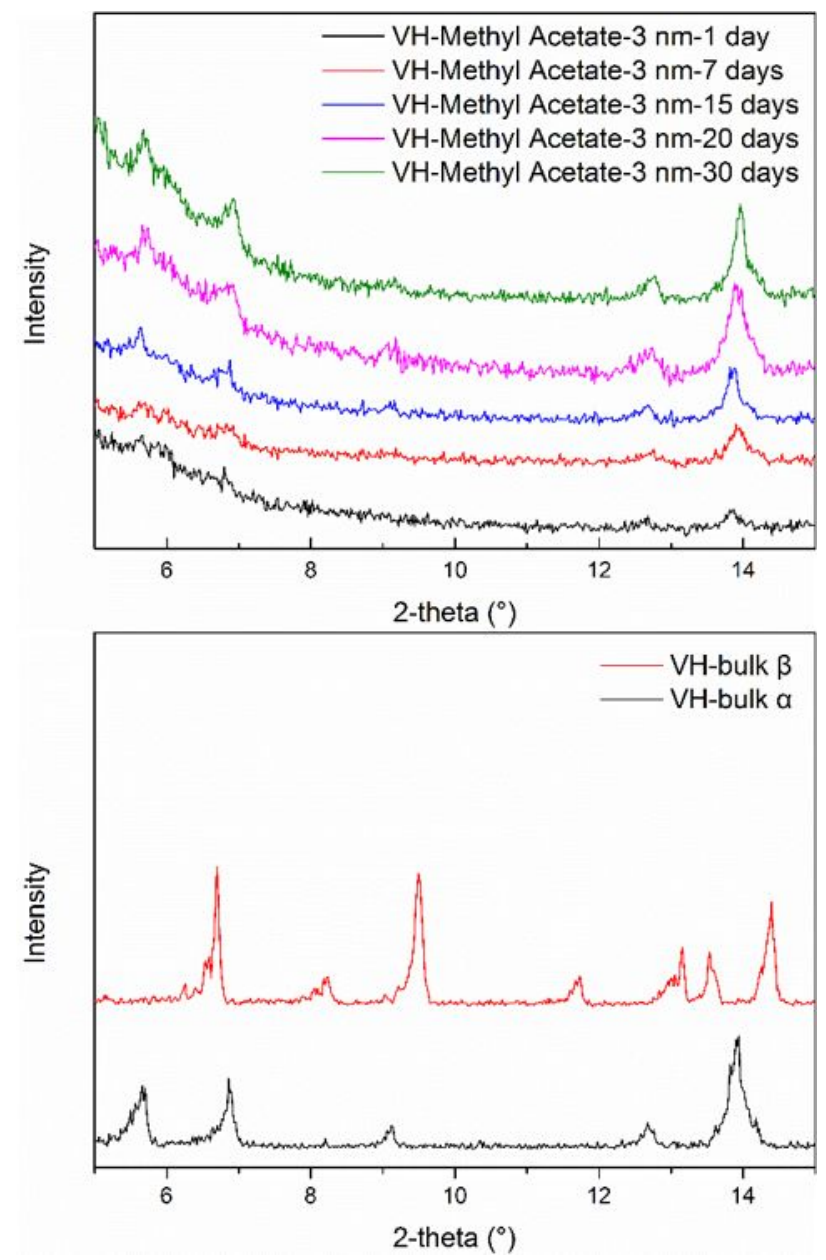

Figure S3. PXRD patterns of confined VH form $\alpha$ under methyl acetate vapor for one month.

As shown, with exposed time increasing, the peak intensity of form $\alpha$ gradually increased, until almost unchanged at the $20^{\text {th }}$ day. This indicates that during the process of exposure, the remaining amorphous VH gradually crystallized. Until the $20^{\text {th }}$ day, complete crystallization was achieved. ${ }^{2}$

6. Solid state of bulk form $\alpha$ and confined form $\alpha$ during dissolution process. 


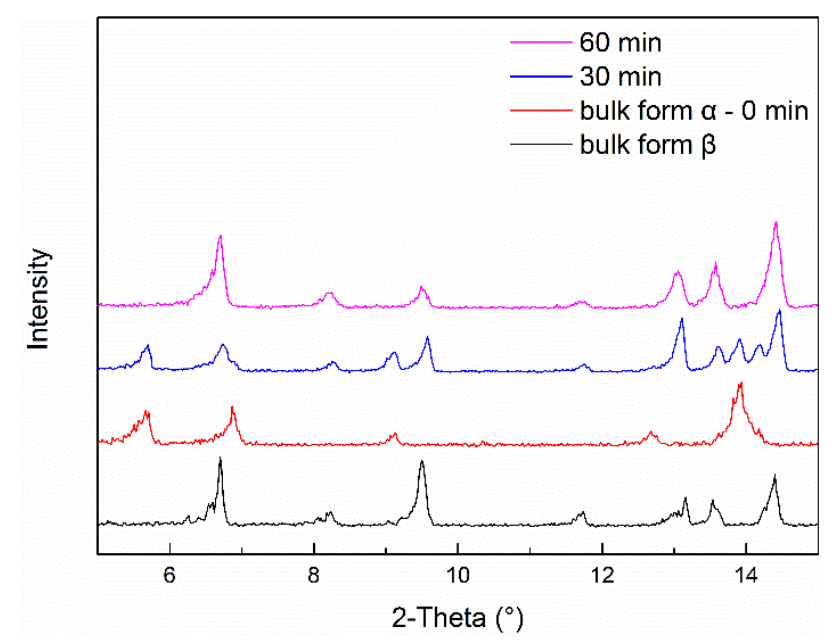

Figure S4. PXRD patterns of suspended particles during the dissolution process of bulk form $\alpha$.

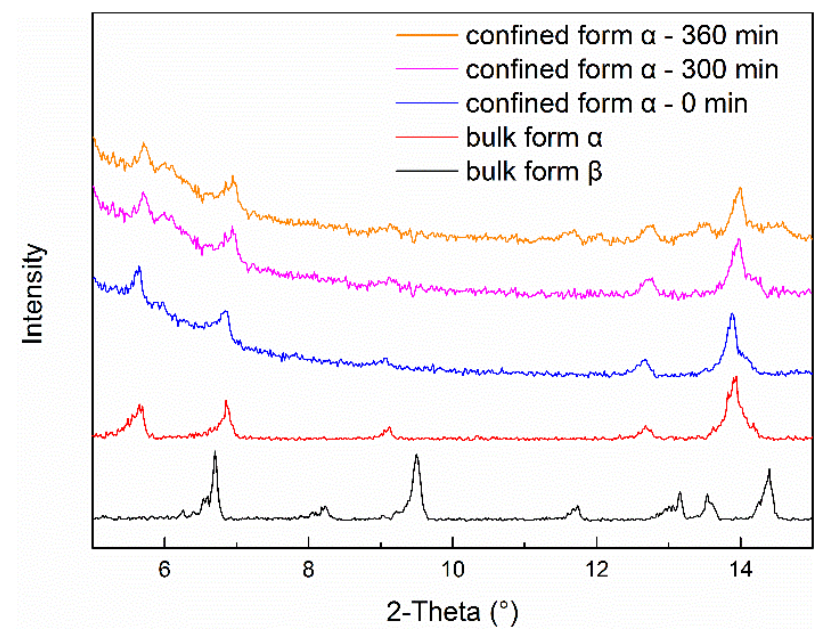

Figure S5. PXRD patterns of suspended particles during the dissolution process of confined form $\alpha$.

\section{Reference:}

1. Cao, Y., Du, S., Ke, X., Xu, S., Lan, Y., Zhang, T., Tang, W., Wang, J., Gong, J. Interplay between Thermodynamics and Kinetics on Polymorphic Behavior of Vortioxetine Hydrobromide in Reactive Crystallization. Org. Process Res. Dev. 2020, 24, 1233-1243. https://dx.doi.org/10.1021/acs.oprd.9b00384.

2. Liu, Y.; Gao, H.; Xu, H.; Ren, F.; Ren, G. Influence of Temperature, Solvents, and Excipients n Crystal Transformation of Agomelatine. Org. Process Res. Dev. 2016, 20, 1559-1565. https://10.1021/acs.oprd.6b00084. 